\title{
Characterization of Density-of-States in Polymer-based Organic Thin Film Transistors and Implementation into TCAD Simulator
}

\author{
Jaehyeong Kim, Jaeman Jang, Minkyung Bae, Jaewook Lee, Woojoon Kim, Inseok Hur, \\ Hyun Kwang Jeong, Dong Myong Kim, and Dae Hwan Kim
}

\begin{abstract}
In this work, we report extraction of the density-of-states (DOS) in polymer-based organic thin film transistors through the multi-frequency $C-V$ spectroscopy. Extracted DOS is implemented into a TCAD simulator and obtained a consistent output curves with non-linear characteristics considering the contact resistance effect. We employed a Schottky contact model for the source and drain to fully reproduce a strong nonlinearity with proper physical mechanisms in the output characteristics even under a very small drain biases. For experimental verification of the model and extracted DOS, 2 different OTFTs (P3HT and PQT-12) are employed. By controlling the Schottky contact model parameters in the TCAD simulator, we accurately reproduced the nonlinearity in the output characteristics of OTFT.
\end{abstract}

Index Terms-Organic thin film transistors (OTFTs), nonlinearity, density-of-states (DOS), P3HT, PQT-12

\section{INTRODUCTION}

Due to flexibility, uniformity, and cost of organic materials, organic semiconductor thin film transistors (OTFT) have been under active development in last years for improved performance as a material for electronic appliances. It is also known to be easy to fabricate the organic semiconductor materials due to their simple structure and low temperature process. Especially,

Manuscript received Apr. 30, 2012; revised Nov. 20, 2012.

School of Electrical Engineering, Kookmin University, Korea E-mail : drlife@kookmin.ac.kr solution-based process allows printing or coating process for the fabrication. Because of these benefits, OTFT is expected to be very promising devices for e-papers and portable/wearable applications.

In this work, report the density-of-states (DOS) in polymer-based organic thin film transistors extracted through the multi-frequency $C-V$ spectroscopy [1]. Extracted DOS is implemented into a TCAD simulator by the C-interpreter method. For the poly (3hexylthiophene) (P3HT) and poly (3,3"' -didodecyl quaterthiophene) (PQT-12) as organic materials (These are p-type semiconductors with bandgap energy $\left(E_{\mathrm{g}}\right)=1.9$ $\mathrm{eV})$, we obtained a consistent transfer $\left(I_{D S^{-}} V_{G S}\right)$ and output $\left(I_{D S}-V_{D S}\right)$ curves with nonlinear characteristics and contact resistance effect. In the simulation, we included the effect of contact resistance by the Schottky contact model parameters on the source and drain electrodes.

\section{STRUCTURE AND FABRICATION OF OTFTS}

For this research, we fabricated coplanar structured thin film transistors as shown in Fig. 1. The fabrication process starts with a sputtered deposition of molybdenum as the gate material on a glass substrate. Then, the silicon dioxide $\left(\mathrm{SiO}_{2}\right)$ is deposited by the plasma enhanced chemical vapor deposition (PECVD) as a gate dielectric. The surface of the gate insulator was treated with a selfassembled monolayer (SAM) of ODTS. Polymer semiconductors were inkjet-printed by a Dimatix printer. Finally, the polymer films were cured at an appropriate temperature $\left(140^{\circ} \mathrm{C}\right.$ for $\mathrm{P} 3 \mathrm{HT}$ and $130^{\circ} \mathrm{C}$ for PQT-12) for 1 hour in a $\mathrm{N}_{2}$ ambient. 


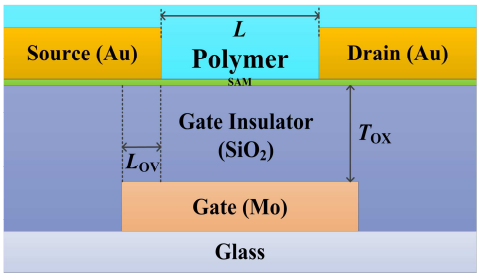

\begin{tabular}{|c|c|}
\hline Parameter & Value \\
\hline $\mathbf{W}[\mu \mathrm{m}]$ & 120 \\
\hline $\mathbf{L}[\mu \mathrm{m}]$ & 12 \\
\hline $\mathbf{T}_{\text {ox }}[\mathrm{nm}]$ & $\mathbf{3 0 0}$ \\
\hline $\mathbf{T}_{\text {active }}[\mathrm{nm}]$ & $\mathbf{3 0}$ \\
\hline
\end{tabular}

Fig. 1. The cross-sectional view and structural parameters of Organic TFTs.

\section{EXPERIMENTAL RESULTS AND DISCUSSION}

We extracted a donor-like DOS $\left(g_{\mathrm{D}}(\mathrm{E})\right)$ through the multi-frequency $C-V$ spectroscopy [1]. The extracted $g_{\mathrm{D}}(\mathrm{E})$ is shown in Fig. 2 and parameters are summarized in Table 1. In order to verify the extracted result, the current-voltage characteristics are simulated by a TCAD simulator from Silvaco. By using a C-interpreter, we implemented the extracted $g_{\mathrm{D}}(\mathrm{E})$ from the OTFTs into the TCAD simulator. For implementation of DOS values
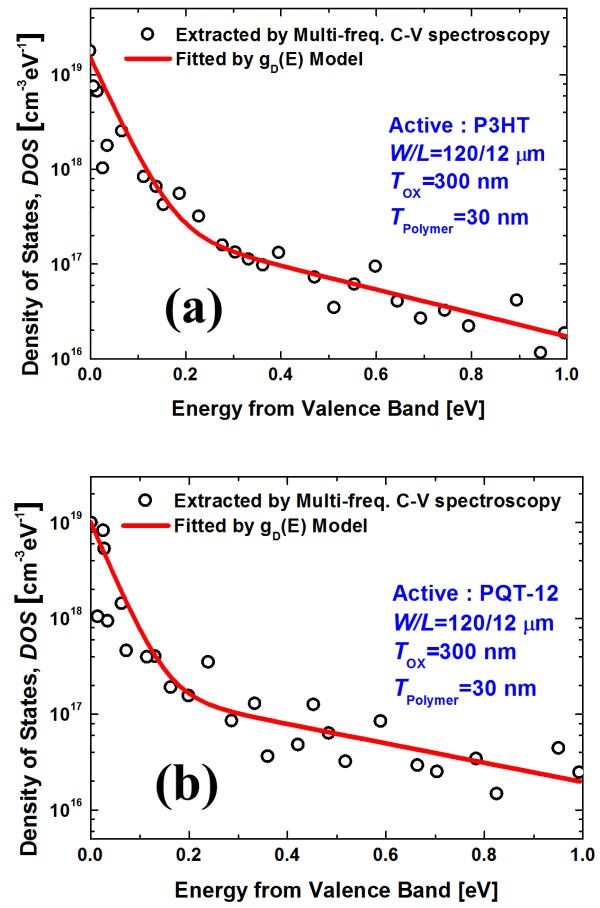

Fig. 2. Extracted $g_{D}(E)$ and models for (a) P3HT, (b) PQT-12.

Table 1. Extracted parameters for $g_{D}(E)$ in OTFTs

\begin{tabular}{|c|c|c|}
\hline Parameter & P3HT & PQT-12 \\
\hline $\mathrm{N}_{\mathrm{TD}}\left[\mathrm{cm}^{-3} \mathrm{eV}^{-1}\right]$ & $1.5 \times 10^{19}$ & $1 \times 10^{19}$ \\
\hline $\mathrm{kT}_{\mathrm{TD}}[\mathrm{eV}]$ & 0.04 & 0.036 \\
\hline $\mathrm{N}_{\mathrm{DD}}\left[\mathrm{cm}^{-3} \mathrm{eV}^{-1}\right]$ & $3 \times 10^{17}$ & $2 \times 10^{17}$ \\
\hline $\mathrm{kT}_{\mathrm{DD}}[\mathrm{eV}]$ & 0.32 & 0.43 \\
\hline
\end{tabular}

into TCAD simulator, we used a well-known DOS model as a superposition of the exponential tail states $g_{\mathrm{TD}}(\mathrm{E})$ and the exponential deep states $g_{\mathrm{DD}}(\mathrm{E}) . g_{\mathrm{D}}(\mathrm{E})$ can be described by

$$
g_{D}(E)=N_{T D} e^{\left(E_{V}-E\right) / k T_{T D}}+N_{D D} e^{\left(E_{V}-E\right) / k T_{D D}}
$$

In the $g_{\mathrm{D}}(\mathrm{E})$, as the energy-dependent donor-like subgap DOS over the forbidden bandgap $\left(E_{V}<\mathrm{E}<E_{C}\right)$, $N_{\mathrm{TD}}\left(N_{\mathrm{DD}}\right)$ and $k T_{\mathrm{TD}}\left(k T_{\mathrm{DD}}\right)$ are the effective density-ofstates and the characteristic energy for the donor-like tail (deep) state in the valence band, respectively.

We note that there is a strong nonlinearity in the output characteristics even at very small drain biases $\left(V_{\mathrm{DS}}\right)$ as shown in Fig. 3(b) and (d). This experimental observation of the nonlinear characteristics is common in OTFTs and caused by the large contact resistance at the source and drain [2]. In order to include this experimental nonlinearity of OTFTs in the TCAD simulator, we considered the contact resistance effect.

For the current-voltage characteristics, the channel carrier mobility and current models are also required for the TCAD simulation [4]. We included the hopping mobility model, Poole-Frenkel mobility model, Schottky contact, and Langevin recombination model. We note that the hopping mobility model is dominated by DOS while the electric field-dependent Poole-Frenkel mobility model describes the movement of charges through traps. The Schottky contact is for a physical description of the poor ohmic characteristics at the source/drain contact while the Langevin model is a well-known recombination model for organic devices.

In order to implement the contact resistance effect in the TCAD simulator of OTFTs, a Schottky contact model is employed for the source/drain contacts. Due to insufficient contact resistance parameters, we controlled the characteristic parameters in the Schottky model described by

$$
J_{S P}=q v_{S U R F P}\left(p_{S}-p_{e q}\right) \exp \left(\frac{\Delta \phi_{b}}{k T}\right)
$$

with $p_{S}$ as a hole concentration at the surface of the contact, $p_{e q}$ as the equilibrium hole concentration, $v_{\text {surfp }}$ as the hole velocity. The image force barrier lowering effect $\Delta \phi_{b}$ is also considered for the Schottky model in 

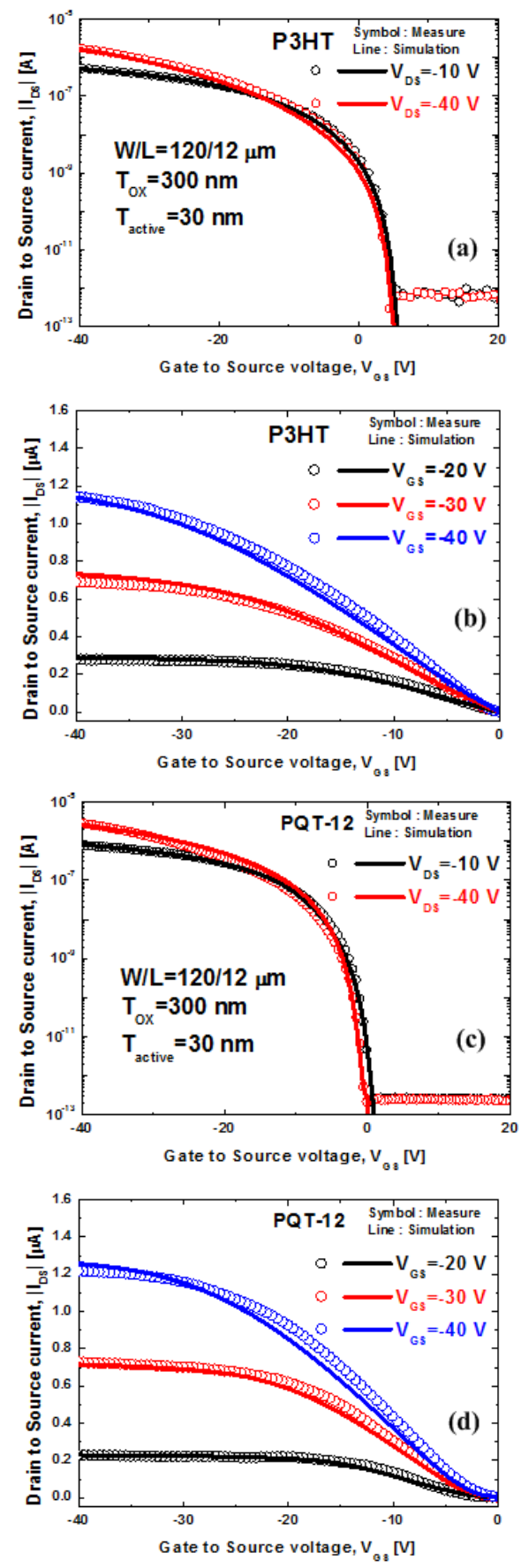

Fig. 3. (a), (c): Transfer curves $\left(V_{\mathrm{DS}}=-10 \mathrm{~V}, V_{\mathrm{DS}}=-40 \mathrm{~V}\right),(\mathrm{b})$, (d): Output curves $\left(V_{\mathrm{GS}}=-20 \mathrm{~V}, V_{\mathrm{GS}}=-30 \mathrm{~V}, V_{\mathrm{GS}}=-40 \mathrm{~V}\right)$ for P3HT, PQT-12 OTFTs. (Open symbol: measurement, solid line: simulation.)

the TCAD simulator. For the n-type metal-silicon Schottky contact, $v_{\text {surf }}$ is typically known to be $v_{\text {surf }}$ $=5.2 \times 10^{5} \mathrm{~cm} / \mathrm{s}$ [3]. Controlling $v_{\text {surf }} \mathrm{x} \exp \left(\Delta \phi_{b} / \mathrm{kT}\right)$ as a fitting parameter, the Schottky contact resistance has been changed to fit to the I-V characteristics especially for the strong nonlinearity in the linear region under a small drain bias.

By implementing the experimentally extracted DOS parameters summarized in Table 1 and the Schottky contact model for the nonlinearity of the output characteristics under a small drain bias into the TCAD simulator, the simulated transfer and output characteristics are shown in Fig. 3 for P3HT and PQT-12 polymer-based OTFTs. We note that the simulated I-V characteristics with DOS parameters obtained from the multi-frequency $C$ - $V$ spectroscopy agree well with the experimental data. This consistent result for the OTFTs with a strong nonlinearity in the linear region is mainly from the inclusion of the Schottky model for the nonlinear contact resistance effect on the source and drain.

\section{SUMMARY}

In this work, we reported experimental characterization of subgap DOS in polymer-based OTFTs and its implementation into the TCAD simulator through a $\mathrm{C}$-interpreter. We used the multi-frequency $C-V$ spectroscopy for the extraction of the DOS in OTFTs. We employed a Schottky contact model for the source and drain resistances to fully reproduce a strong nonlinearity with proper physical mechanisms in the output characteristics even under a very small drain biases. We employed 2 different OTFTs (P3HT and PQT-12) for experimental verification of the model and extracted DOS. By controlling the Schottky contact model parameters in the TCAD simulator, we accurately reproduced the nonlinearity in the output characteristics of OTFTs. We expect that this approach is very effective and useful for estimation of the electrical properties and practical application of high performance OTFTs to circuits and systems through a TCAD simulation.

\section{ACKNOWLEDGEMENTS}

This work was supported by the National Research Foundation(NRF) grant funded by the Korea government (MEST) (No. 2010-0013883 and 2009-0080344) and the CAD softwares were supported by Silvaco and IC Design Education Center (IDEC).

The devices were supported by Samsung Advanced Institute of Technology (SAIT). 


\section{REFERENCES}

[1] J. Jang, et al., "Extraction of the sub-bandgap density-of-states in polymer thin-film transistors with the multi-frequency capacitance-voltage spectroscopy," Appl. Phys. Lett. Vol.100, No.13 (2012), p.133506 133506-5

[2] G. B. Blanchet, et al., "Contact resistance in organic thin film transistors," Appl. Phys. Lett. Vol. 84, No.2 (2004), p.296 298

[3] C. R. Crowell, et al., "CURRENT TRANSPORT IN METAL-SEMICONDUCTOR BARRIERS," Solid-State Electronics, Vol.9, (1966), p.1035 1048.

[4] ATLAS Device simulation software User's Manual, SILVACO International.



Jaehyeong Kim received the B.S. degree in electrical engineering from the Kookmin University, Seoul, Korea, in 2011, where he is currently working toward the M.S. degree at the Department of Electrical Engineering under the supervision of Prof. D. M. Kim and Prof. D. H. Kim. His current research mainly focuses on amorphous oxide / organic semiconductor TFTs and their device physics and modeling.

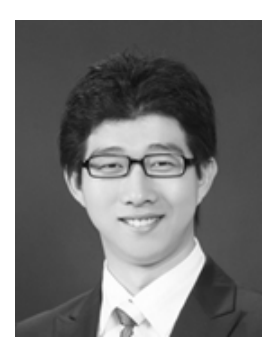

Jaeman Jang received the B.S. and M.S. degree in 2009 and 2011, respectively, from Kookmin University, Seoul, Korea, where he is currently working toward the Ph.D. degree in electrical engineering. During his M.S. studies, he has worked on modeling, characterization and design of nano-scaled memory device including capacitorless $1 \mathrm{~T}$ DRAM and nano-crystal flash memory device. His current research interest includes design, fabrication, characterization, and modeling of oxide thin film transistors and organic thin film transistors.

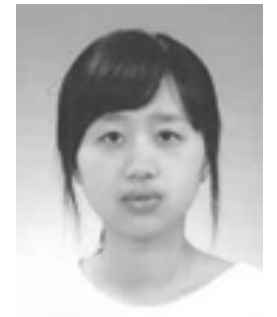

Minkyung Bae received the B.S. and M. S. degrees in electrical engineering from the Kookmin University, Korea, in 2010 and 2012, respectively. She is currently with Samsung Electronics Company, Ltd., Korea. Her research interests are nanoCMOS

devices and TFTs.

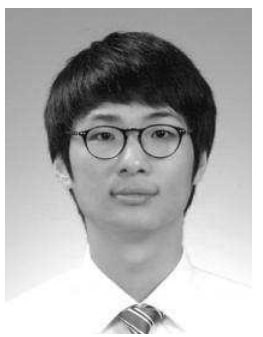

Jaewook Lee received the B.S. degree in electrical engineering from the Kookmin University, Seoul, Korea, in 2012, where he is currently working toward the M.S. degree at the Department of Electrical Engineering under the supervision of Prof. D. M. Kim and Prof. D. H. Kim. His current research mainly focuses on organic semiconductor TFTs and their device physics.

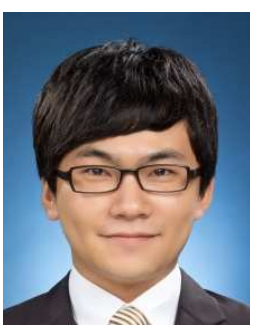

Woojoon Kim received the B.S. degree in electrical engineering from the Kookmin University, Seoul, Korea, in 2011, where he is currently working toward the M.S. degree at the Department of Electrical Engineering under the supervision of Prof. D. M. Kim and Prof. D. H. Kim. His current research mainly focuses on amorphous oxide semiconductor TFTs and their device physics.

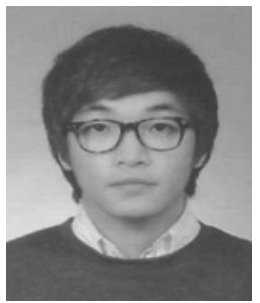

Inseok Hur received the B.S. degree in electrical engineering from Kookmin University, Seoul, Korea, in 2011, where he is currently working toward the M.S. degree in the Department of Electrical Engineering under the supervision of Prof. D. M. Kim and Prof. D. H. Kim. His current research mainly focuses on amorphous oxide semiconductor thin-film transistors and their device physics. 


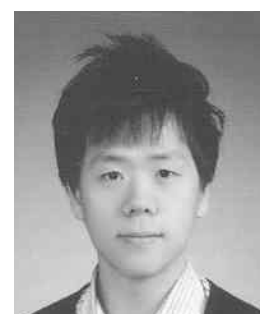

Hyun Kwang Jeong received the B.S. and M. S. degrees in electrical engineering from the Kookmin University, Korea, in 2010 and 2012, respectively. $\mathrm{He}$ is currently with Magna Chip company, Korea. His research interests are nano CMOS

devices.

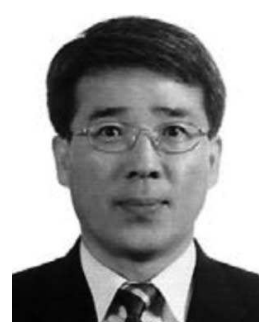

Dong Myong Kim (S'86-M'88) received the B.S.(magna cum laude) and M.S. degrees in electronicsengineering from Seoul National University, Seoul,Korea, in 1986 and 1988, respectively, and the Ph.D. degree in electrical engineering from the University of Minnesota, Twin Cities, in 1993.From February 1988 to August 1989, he was with the Division of Electronic Engineering at Korea Institute of Science and Technology (KIST), Seoul, Korea, where he worked on the characterization and modeling of microwave devices and integrated circuits. Since March 1993, he has been with the School of Electrical Engineering, Kookmin University, Seoul, Korea. He is also serving as a Special Lecturer for Semiconductor Physics and Devices at Samsung Electronics Co., Hwasung, Korea since April 2002. His current research interests include design, fabrication, characterization, and modeling of nanostructure silicon devices, thin film transistors, biosensors, III-V compound semiconductor devices, volatile and nonvolatile memories, and CMOS RF circuits.

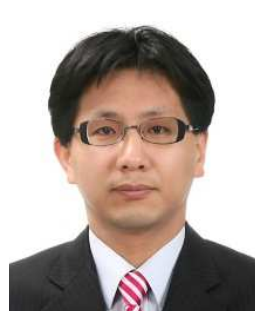

Dae Hwan Kim (M'08) received the B.S., M.S., and Ph.D. degrees in electrical engineering from Seoul National University, Seoul, Korea, in 1996, 1998, and 2002, respectively. From 2002 to 2005, he was with Samsung Electronics Company, Ltd., Kyung ki-Do, Korea, where he contributed to the design and development of 92-nm DDR DRAM and 80-nm DDR2 DRAM. In 2005, he joined the School of Electrical Engineering, Kookmin University, Seoul, where he is currently an Associate Professor. He has authored or coauthored more than 170 research publications and patents. His current research interests are nanoscale CMOS devices and integrated circuits, metal oxide and organic thin-film transistors, biosensors devices, exploratory logic and memory devices, energyefficient nano-ICs, and Si quantum devices. He has also worked on the characterization, modeling, and circuit design for reliabilities of CMOS devices, thin-film transistors, display, biosensors, and neuromorphic systems. He is a member of IEEE, Society for Information Display, and Institute of Electronics Engineers of Korea. 